\title{
Lumen
}

Selected Proceedings from the Canadian Society for Eighteenth-Century Studies

\section{La fin de la notion de passion. Réflexions sur la mort d'une notion}

\section{Jean-Pierre Cléro}

Volume 23, 2004

URI : https://id.erudit.org/iderudit/1012186ar

DOI : https://doi.org/10.7202/1012186ar

Aller au sommaire du numéro

Éditeur(s)

Canadian Society for Eighteenth-Century Studies / Société canadienne d'étude du dix-huitième siècle

ISSN

1209-3696 (imprimé)

1927-8284 (numérique)

Découvrir la revue

Citer cet article

Cléro, J.-P. (2004). La fin de la notion de passion. Réflexions sur la mort d'une notion. Lumen, 23, 39-73. https://doi.org/10.7202/1012186ar

Copyright @ Canadian Society for Eighteenth-Century Studies / Société canadienne d'étude du dix-huitième siècle, 2004
Ce document est protégé par la loi sur le droit d'auteur. L'utilisation des services d'Érudit (y compris la reproduction) est assujettie à sa politique d'utilisation que vous pouvez consulter en ligne.

https://apropos.erudit.org/fr/usagers/politique-dutilisation/ 


\section{La fin de la notion de passion. Réflexions sur la mort d'une notion}

«Somme toute, en ce monde desrayé, rien ne debvant, rien de prestant, rien ne empruntant, vous voirez une conspiration plus pernicieuse que n'a figuré Aesope en son Apologue. Et perira sans doubte : non perira seulement, mais bien tost perira, feust ce Aesculapius mesmes. Et ira soubdain le corps en putrefaction : l'ame toute indignée prendra course à tous les Diables, apres mon argent.»

Rabelais $^{1}$

«Il faut que tout se paie : l'opulence par le travail, les faveurs par la flatterie. Voudrais-je ne pas payer le prix et pourtant avoir l'avantage ?» Hume $^{2}$

«Se sacrifier à ses passions, passe ; mais à des passions qu'on n'a pas ! $O$ triste dix-neuvième siècle !»

Girodet $^{3}$

Je voudrais présenter, dans le cadre de ce colloque, un fragment de recherche sur un problème général qui commence à faire l'objet d'une enquête, de la part de chercheurs enseignant dans des universités québécoises et françaises, touchant l'explication du changement de la notion de passion, d'une époque où elle paraît avoir une consistance ontologique solide, comme c'est encore le cas au XVII ${ }^{e}$ siècle, même si elle varie beaucoup d'un auteur à l'autre, à une époque, que je situe vers la fin du XVIII ${ }^{\mathrm{e}}$ siècle et au début du XIX ${ }^{\mathrm{e}}$ siècle, où la notion même de passion est considérée comme une entité fictive, d'assez piètre valeur

1 Chap. 3, «Comment Panurge loue tous les debteurs et emprunteurs», Le Tiers Livre.

2 Le Sceptique, 7 e réflexion.

3 Citation de Stendhal en exergue du chapitre 29 du tome 2 de Le Rouge et le noir. 
idéologique et sans plus aucune portée scientifique. Certes, l'établissement de ce fait est loin d'être avéré : il me faudra le présupposer. Je me contenterai donc, en guise de démonstration et afin de rendre plausible la présente recherche, de quelques remarques préliminaires.

Certes, dès les années 1650, on note une différence entre la position de Descartes, qui traite les passions comme résultant, dans leur diversité, de l'union substantielle de l'âme et du corps, et celle de Pascal, qui en tient l'unité pour plus nominale et à qui il arrive d'étiqueter les passions, non pas sous des noms communs, comme amour, haine, désir, etc., mais sous le nom propre de Babylone 4 . Mais s'il met davantage l'accent sur l'unité seulement structurelle de la passion, Pascal n'en demande pas moins à chacun, à plusieurs reprises, de travailler à diminuer ses passions ${ }^{5}$, comme si elles étaient un matériau que l'on pût ouvrager. Il est clair qu'un tel précepte, un siècle et demi plus tard, apparaîtra à Bentham comme un slogan à peu près vide de contenu, qui n'a plus aucun sens direct et qui, s'il ne doit pas être abandonné, requiert une radicale traduction ou réversion. Le discours de la passion n'est plus qu'une rhétorique destinée à couvrir des éléments structurels beaucoup plus opérants et sur lesquels on peut réellement agir, le sentir passionnel n'étant que le fumet évanescent de ceux-ci, susceptible d'égarer ceux que $l^{\prime}$ 'on a intérêt à berner. Dans les premières décennies du XVIII siècle et au début du XIX ${ }^{\mathrm{e}}$, on n'imagine plus rien désigner de réel, du moins directement, en parlant de passions, comme on pouvait le croire au XVII siècle : le mot ne paraît plus désigner indirectement que quelque chose de tout autre. Quoi?

Il est clair que, si les traités, les essais, les dissertations, les chapitres et les livres à l'intérieur des grands ouvrages de philosophie et de théologie abondent à l'âge classique sur le thème des passions, la veine parait s'être assez brusquement tarie ; les traités de psychiatrie, les essais d'économie - les réflexions sur le «commerce», comme on disait alors

4 Les Pensées, p. 716: «Qu'on voie si ce plaisir est stable ou coulant; s'il passe, c'est un fleuve de Babylone» ; p. 253 : «Après [la] mort [de Jésus-Christ], saint Paul est venu apprendre aux hommes que toutes ces choses étaient arrivées en figure, que le royaume de Dieu ne consistait pas en la chair, mais en l'esprit, que les ennemis des hommes n'étaient pas les Babyloniens, mais leurs passions, etc.», dans Michel Le Guern (édit.), CEuvres complètes, Paris, NRF Gallimard, 2000, vol. II. Toutes les citations de Pascal font référence à cette édition.

5 Les Pensées, p. 397 : «Apprenez au moins que votre impuissance à croire vient de vos passions, puisque la raison vous y porte et que néanmoins vous ne le pouvez. Travaillez donc, non pas à vous convaincre par l'augmentation des preuves de Dieu, mais par la diminution de vos passions». 
—, les histoires — au sens où Hume trace L'Histoire d'Angleterre -, les traités de philosophie du droit - de jurisprudence, selon le terme en usage - les ouvrages de politique, se sont substitués aux discours tenus sur les passions, les rendant caduques et obsolètes. Ainsi, en 1759, dans sa Théorie des sentiments moraux, A. Smith dira que «les systèmes de droit positif [...] méritent la plus grande autorité en tant qu'ils sont les registres des sentiments du genre humain dans les différentes époques et les différentes nations ${ }^{6}{ }^{6}$, et suggérera, non plus seulement qu'il faut connaître le fonctionnement des passions pour comprendre les règles de droit lorsqu'elles régissent les contrats, les mariages, les châtiments, les héritages et toutes sortes d'autres institutions, mais aussi, à l'inverse, que la façon dont le droit s'organise est une structuration des façons de sentir, sans que les hommes n'y prennent toujours garde. Étrange aveu d'un auteur qui, à la fin d'un livre sur les sentiments moraux, renvoie au Cours de jurisprudence, comme étant son véritable fondement; les sentiments et les passions se dissolvant dans le simple jeu des apparences $\mathrm{du}$ fonctionnement des codes et de ses changements. On reçoit la confirmation de ce renversement quand on lit le Cours de jurisprudence lui-même, bien entendu, mais aussi La Richesse des nations, dont la psychosociologie de figures comme celle du parvenu ${ }^{7}$, du marchand, avaricieux par nature, de la victime de l'exode $\mathrm{rural}^{8}$, s'explique par les possibilités juridiques certes, mais aussi par les circuits du commerce et de la production qui sont les réels moteurs des affects, à moins que ce ne soit, plus profondément, comme il est suggéré dès les premières lignes de La Richesse des nations, par des éléments plus symboliques de l'échange et de la circulation : je veux parler du langage ${ }^{9}$. On assiste donc, tout le long du XVIII ${ }^{\mathrm{e}}$ siècle, à un effort de substitution partielle au discours traditionnel des passions par toutes sortes de discours dont chacun se garde bien de dire quel est ou quel sera ultimement le plus

6 Adam Smith, Théorie des sentiments moraux, Paris, PUF, 1999, p. 453.

7 Adam Smith, Lectures on Jurisprudence, Ronald L. Meek, David D. Raphaël and Peter Stein (édit.), Oxford, Clarendon Press, 1978, p. 321, 402.

8 Adam Smith, Enquête sur la nature et les causes de la richesse des nations, trad. Paulette Taieb, Paris, PUF, 1976, p. 466-7, 762.

9 Adam Smith, Enquête sur la nature: «Il n'entre pas dans notre présent propos de rechercher si cette propension est un des principes innés de la nature humaine, dont on ne peut rendre davantage compte; ou si, comme cela semble plus probable, elle est la conséquence nécessaire des facultés de raison et de parole», vol. I, p. 15. [C'est nous qui soulignons cet aspect qui s'accommode mal d'une lecture «naturaliste» d'Adam Smith]. 
explicatif $^{10}$; je dis partielle, car les auteurs ne vont pas jusqu'à une élimination du discours de la passion, qui subsiste comme une sorte $\mathrm{d}$ 'accompagnement auquel on a recours à divers titres dans les explications, quoiqu'il soit déjà frappé d'une sorte de minorité ontologique. Le travail de Bentham est très différent par sa radicalité ; comme Hilbert montrera sur le terrain des mathématiques, dans ses Fondements de la géométrie, qu'il ne rencontre pas les théorèmes classiques de Pascal et de Desargues, Bentham fait une véritable réduction logique de la notion de passion, à laquelle il dénie toute effectivité, et il ne la reconstruit que pour expliquer comment une réalité se trouve masquée par son jeu fictif et fallacieux. On peut bien encore parler de passion - n'y a-t-il pas aujourd'hui même pléthore de littérature philosophique et historienne sur la question? - mais comme d'une notion révolue, à laquelle on ne reviendra plus pour expliquer fondamentalement les choses ; pas plus que le théorème de Desargues ne règle fondamentalement les espaces modernes. Mais comment cette notion de passion disparaît-elle ? Comment se résorbe-t-elle? Comment «meurt»-t-elle, si l'on veut bien donner à cette question un sens différent de "comment la réfute-t-on?», car une notion peut longtemps survivre à sa réfutation?

Comme, dans le temps de cet exposé, il est impossible de considérer tous les aspects de cette résorption de la notion de passion, je m'en tiendrai à un seul, dans la mesure où il se laisse isoler : il s'agit de considérer comment la structure de paiement qui paraissait sous-tendre un grand nombre de passions à l'âge classique est graduellement reconnue comme indépendante des passions qui l'accompagnent au point qu'elles se résorbent en elle et dans ses calculs. La question devient donc: qu'est-ce que disparaître, pour une notion comme la passion, quand s'y substitue la trame des paiements, de concert avec d'autres fils que, par décision méthodique, je ne considérerai pas ici.

Plutôt que de traiter la question en historien, ce qui serait peut-être la meilleure approche, mais probablement indéfinie par le nombre terrifiant d'œuvres qu'il faudrait consulter, je veux esquisser la direction générale d'un mouvement, tel qu'il peut nous apparaître aujourd'hui, sans tenir compte de sa fractalisation historique, et tracer, en une sorte $\mathrm{d}^{\prime}$ histoire philosophique - faute de mieux $\mathrm{x}^{11}$ - les quatre moments

10 Curieusement, il arrive qu'un auteur estime plus fondamental un discours qu'il n'a pas lui-même choisi de faire, jouant en quelque sorte sur deux tableaux et sacrifiant à une sorte de double vérité.

11 Car il s'agit d'une histoire idéale, forcément simplificatrice. 
fondamentaux d'un déclin, lesquels ne correspondent pas nécessairement avec une datation empirique. Vient d'abord, avec Pascal, le moment «moral» de la décomposition de la notion de passion; on trouve ensuite, chez Hume, dans un effort renouvelé pour dire ce que sont les passions, un discours clivé, qui ne donne lieu qu'à des propositions antithétiques ; apparaît, si tant est qu'on puisse lui assigner une date, l'approche cynique de la question, qui est une réduction de la notion : la passion $n^{\prime}$ est que le paiement dont elle est le travestissement. C'est avec Bentham que nous trouvons la résolution véritable et définitive de la passion, permettant d'assimiler les retours de la notion à des combats d'arrière-garde, sans grande portée, sinon celle de nous assurer toujours davantage qu'elle n'existera jamais plus de la même façon qu'à l'âge classique.

\section{Le moment pascalien}

Il faut donc partir de Pascal, toujours attentif à l'essence «structurale» des passions et plutôt prêt à dénoncer qu'à adopter l'approche substantielle de Descartes. Certes, il rappelle bien que sentir et imaginer qu'on sent ne reviennent pas au même ; il n'empêche qu'il est toujours possible, et que c'est même le mode le plus ordinaire du sensible, d'imaginer qu'on sent $^{12}$. Dès lors, $c^{\prime}$ est, sous couvert de cette impression fallacieuse, tout un système et tout un circuit qui œuvrent, à l'insu de la volonté consciente des hommes ${ }^{13}$. Ce ne sont pas les passions qui intéressent Pascal, c'est, comme l'a vu Scheler ${ }^{14}$, leur logique. La structure des relations entre les hommes sous-tend leurs sentiments qui, à la fois, l'expriment et se trouvent en rapport de tension avec elle. Soit le court fragment des Pensées que chacun connaît et que je veux prendre pour le fil rouge du présent «moment» : «... trop de bienfaits irritent. Nous voulons avoir de

12 Les Pensées, p. 756 : «Les hommes prennent souvent leur imagination pour leur cœur».

13 Qu'il soit parfaitement clair que Pascal n'est pas pris ici comme le premier auteur qui envisage les aspects structurels de la passion dans la substitution à ladite passion. On trouve, dans le livre même de la Rhétorique d'Aristote, bien des passages qui montrent que des passions vont de pair avec des éléments structuraux, qu'ils soient politiques, économiques, institutionnels. C'est surtout vrai de la honte et de l'impudence. Pascal présente toutefois, sur Aristote, l'avantage de pousser beaucoup plus loin, grâce à ses aspects antinaturalistes, l'esquisse des circuits structuraux.

14 Max Scheler, Le Formalisme en éthique et l'éthique matériale des valeurs, Paris, NRF Gallimard, 1955, p. 86 : "Comme le dit très bien Blaise Pascal, il existe un «ordre du cœur» ou une «logique du cœur» qui sont $a$ priori». 
quoi surpasser la dette ${ }^{15}{ }$. Suit une citation de Tacite que Pascal a probablement trouvée dans les Essais de Montaigne : «Les bienfaits sont agréables tant qu'ils paraissent pouvoir être payés de retour. S'ils dépassent de beaucoup ce pouvoir, au lieu de reconnaissance, nous les payons de haine ${ }^{16}{ }^{\prime}$. On peut gager qu'une personne qui agit de la sorte le fait sans remords et se sent parfaitement justifiée de se conduire avec ce qui parait, de l'extérieur, ingratitude ${ }^{17}$; c'est que le sentiment, tel qu'il est senti ou tel qu'on se figure le sentir, ne fait que refléter, comme dans un miroir, c'est-à-dire moyennant une inversion, tout ce qui se fait réellement, en l'occurrence la réalité du paiement. Quiconque rend un service à une personne, en lui donnant quelque chose qu'elle ne pourra pas rendre, l'humilie ; puisqu'il lui inflige le désagrément d'être dépendante malgré elle de l'élever ou de la gratifier en lui intimant un retard ou une dette à son égard. Il n'y a sans doute pas de réception innocente ; il n'y a pas davantage de donation innocente. Même si elle est imaginaire, il suffit que l'humiliation soit vécue comme existante par la personne qui en fait les frais pour qu'elle ait l'effet d'une humiliation réelle. Le seul moyen de remboursement qui s'offre à la personne ainsi humiliée, c'est de demander le dédommagement de l'humiliation, qui est infini ou, du moins, qui enclenche un processus interminable, car ce ne sont pas les choses et les services qui sont en jeu, mais les personnes mêmes, fussent-elles des invocations fictives. Tout payeur ou tout débiteur paie, qu'il le veuille ou non, de sa personne ${ }^{18}$. L'ingratitude, qu'on se sente ou non coupable d'y sacrifier, est inscrite dans le circuit même des paiements, qui est une logique autrement plus efficace que tout ce qu'on peut en sentir. Le paiement implique toujours que le non-remboursement devienne luimême le remboursement. Tout l'art de celui qui rembourse est de payer avec l'argent même de celui qui a déjà payé, en le forçant à surpayer ${ }^{19}$.

15 Les Pensées, frag., p. 185.

16 «Beneficia eo usque laeta sunt dum videntur exsolvi posse ; ubi multum antevenere, pro gratia odium redditur», Tacite, Annales, liv. 4, chap. 18, cité par Montaigne, Essais, liv. 3, chap. 8 .

17 Ainsi en va-t-il de Simon, dans les Provinciales, qui, selon Pascal, ne se sait pas coupable de simonie (IX ${ }^{\mathrm{e}}$ lettre).

18 Chacun connaît la remarque du Prince de Machiavel : «La nature des hommes est de s'attacher aussi bien par les bienfaits qu'ils font que par ceux qu'ils reçoivent» (p. 110-111).

19 Principe élémentaire dont Machiavel fait la théorie dans Le Prince, chapitre 16 : «dépenser ce qui est à autrui n'ôte rien à ta réputation mais l'accroît; dépenser ce qui est à toi est la seule chose qui te nuit». 
L'ingratitude est l'essence du circuit des paiements et celui qui la ressent comme celui qui se la masque - et cette dissimulation n'est pas très difficile puisqu'elle est conforme à l'exigence du circuit - ne sait généralement pas qu'il entre et fait entrer dans un interminable circuit. Le sentiment d'ingratitude, par l'impression de dissension qu'il suscite, cache assez bien la machine à lier les hommes qui, pourtant, le provoque mais fonctionne selon des lois qui paraissent ignorer tant les affects que les volontés.

C'est très exactement ce que veut dire Pascal lorsqu'il montre, en quelques fragments des Pensées, que le seul fonctionnement de la concupiscence, sans qu'aucune parcelle de charité ne s'en mêle, fabrique les lois et les gouvernements des sociétés ${ }^{20}$ en évitant, autant que faire se peut, les guerres civiles. Certes, la justice qui en résulte n'a rien à voir avec la charité chrétienne que Pascal lui oppose, mais il admire qu'elle crée un lien puissant qui suffit à organiser les sociétés. Les hommes ne s'aiment, ni eux-mêmes, ni entre eux, d'un véritable amour, mais le jeu des images et la pseudo-production de droit qui résulte des passions ${ }^{21}$ suffisent à les cimenter fortement. Simplement il est bon que la plupart des hommes n'en aient pas conscience et l'on se lance dans une mauvaise besogne de demi-habiles lorsqu'on veut les rendre tous conscients de la vanité des images, qu'ils voudront détruire en une fureur iconoclaste, sans bien se rendre compte que toute cette vanité a plus de prix que la guerre. Si fondamentalement déprécié soit-il comme figmentum malum, le leurre est paradoxalement valorisé par Pascal, car même à titre de fausse monnaie, il n'en rend pas moins le service d'unifier la société, du moins tant qu'elle n'est pas détruite par contradictions internes ou tant que les certitudes profondes et inconscientes ne sont pas perturbées par l'effet désastreux de quelque événement.

20 Les Pensées, frag., p. 97, 109 ("Grandeur de l'homme dans sa concupiscence même, d'en avoir su tirer un règlement admirable et en avoir fait un tableau de la charité»), p. 196 («Tous les hommes se haïssent naturellement l'un l'autre. On s'est servi comme on a pu de la concupiscence pour la faire servir au bien public. Mais ce n'est que feindre et une fausse image de la charité, car au fond ce n'est que haine»), p. 197 («On a fondé et tiré de la concupiscence des règles admirables de police, de morale et de justice. Mais, dans le fond, ce figmentum malum n'est que couvert. Il n'est pas ôté»).

21 Pascal a parfaitement vu comment chaque passion produisait son droit naturel. L'amoureux fait payer à l'autre le fait de lui avoir plu et d'avoir été séduit. Le destin de toute passion consiste à rendre l'autre débiteur, à faire qu'il nous doive quelque chose et à exiger qu'il s'acquitte de ces pseudo-devoirs. Ces pseudo-devoirs, qui sont ceux du droit naturel, cimentent néanmoins réellement les hommes entre eux. 
La plupart du temps, nous supportons parfaitement la contradiction : ainsi feignons-nous de croire que nos sentiments naturels - du moins certains d'entre eux, comme le sentiment qui lie les pères aux fils et aux filles - tout en nous comportant comme si on ne les croyait pas tels puisqu'on déploie, pour maintenir ces liens, toutes sortes d'activités qui laissent penser qu'on les croit artificiels ${ }^{22}$. Chacun sait intimement que la nature est un second artifice, mais chacun préfère aussi s'imaginer l'artifice comme s'il était naturel, par une seconde nature. Dans le circuit des paiements qui préside à l'ingratitude, tout est vu à l'envers : celui qui paie croit en finir avec les paiements, alors même qu'il enclenche le système ; celui qu'il rend dépendant le rend, en retour, lui-même dépendant en lui faisant surpayer ce qu'il a donné comme une dette supérieure à celle qu'il couvrait ; ce que les acteurs voient du paiement n'est qu'un petit segment d'un immense circuit qu'ils enclenchent sans qu'ils sachent jamais qui l'a enclenché, où sont les limites de ceux qu'il concerne et quand il s'achèvera. Le sentiment implique un voile et une perspective resserrée sur la totalité ; il est solidaire d'une dynamique dont ceux qui le ressentent n'aperçoivent pas les vrais ressorts, mais dont certains événements font soupçonner l'existence.

D'ailleurs, fondamentalement les hommes ne croient pas à la vérité de leurs sentiments ; ils se figurent seulement - et c'est là leur point de naïveté - que les autres ont des sentiments réels et ils en attendent de leur part. Ainsi la réalité de l'amour est-elle dans la séduction, dans la volonté de reproduire au sein de la vie réelle ce qu'on a vu sur des scènes imaginaires et elle réside, par conséquent, dans une sorte de passage à $l^{\prime}$ acte de nos fantasmes ${ }^{23}$. Si bien qu'au lieu de vivre ils sont constamment en train d'espérer de vivre ${ }^{24}$, la fiction ayant autant, voire plus, de force que la réalité même. On aura noté que, dans l'analyse qu'il fait de quelques passions, de l'ingratitude en particulier, Pascal met en avant le plaisir, la douleur et une articulation de ceux-ci comportant un jeu temporel qui relie réalité et fiction dans la même structure.

22 Les Pensées, frag., p. 117 : «Les pères craignent que l'amour naturel ne s'efface. Quelle est donc cette nature sujette à être effacée ? La coutume est une seconde nature qui détruit la première. Mais qu'est-ce que nature ? Pourquoi la coutume n'est-elle pas naturelle ? J'ai grand peur que cette nature ne soit elle-même qu'une première coutume, comme la coutume est une seconde nature».

23 Ce qui engage Pascal à condamner la comédie. Les Pensées, frag., p. 640.

24 Les Pensées, frag., p. 43. La procrastination est, chez Pascal, l'un des modes existentiels nécessaires à la vie de nos passions. Un très grand nombre de nos passions sont telles que nous nous donnons à les sentir plus tard, sans que nous ne les sentions jamais. 
Ce dernier point ne doit pas être oublié car on tient avec lui l'un des principaux éléments qui feront imploser le système des passions et le discours sur elles ; mais il faut encore faire deux autres remarques. La première est qu'on aurait grand tort de ne prendre que métaphoriquement l'analogie du fonctionnement de l'ingratitude avec la circulation de l'argent. Certes, le circuit de l'argent peut s'analyser comme une passion (Qui paie ? À qui ? Comment ? Avec quoi ? Pour quoi faire? Pour quelle raison ?) : on peut poser aux paiements les mêmes questions qu'aux passions. C'est ce qui fait qu'on peut si bien énoncer les passions dans la langue de l'argent : les renversements auxquels est soumise la passion peuvent se décrire dans une logique commerciale de prix ; $c^{\prime}$ est ce qui fait aussi qu'on ne paie jamais ce qu'on croit payer, pas plus que $l^{\prime}$ 'objet de ces passions est un objet véritable. Il en va de même pour tous les autres pôles de la structure. Mais l'affinité va plus loin et se transforme en un véritable entrelacement lorsque l'on saisit que ce que l'on paie est moins des marchandises que du plaisir et de la douleur; que c'est toujours une relation de sujet à objet qui est payée, plutôt que la relation des choses entre elles pour laquelle elle se donne; que cette relation même du sujet et de l'objet a elle-même son prix, indéfiniment. Les multiples façons selon lesquelles le circuit des paiements se laisse lire s'articulent avec les multiples façons dont les affects se laissent eux-mêmes déchiffrer, y compris par le sentir même de ceux qui les ressentent. Il est, chez Pascal, une conception de l'argent parfaitement commensurable avec ce qu'il dit des passions. Comment aurait-il pu échapper au calculateur des partis que l'espérance même a son prix ? L'argent et les passions s'entrelacent parce qu'il y a un argent de l'argent, une passion de la passion et que l'un et l'autre s'articulent au mieux dans un jeu de fictions dont le calcul des probabilités permet d'esquisser ou $\mathrm{d}^{\prime}$ entrevoir la logique ${ }^{25}$. Cet entrelacement signifie sans doute que le soubassement des passions est de nature économique, mais aussi que l'économie n'a pas de sens sans les interminables complications de plaisir et de douleur que les classiques ont appelées passions.

Mais il est une autre observation à faire : la conception pascalienne des passions est ouvertement morale $^{26}$. Si la structure des passions est

25 «Ce n'est pas qu'on s'imagine que la vraie béatitude soit d'avoir l'argent qu'on peut gagner au jeu [...] : on n'en voudrait pas s'il était offert». «Donnez-lui tous les matins l'argent qu'il peut gagner chaque jour, à la charge qu'il ne joue point : vous le rendrez malheureux». Sans compter que l'on peut différer des paiements comme on procrastine des affects.

26 C'est dans les Provinciales qu'elle mérite le mieux cette qualification ; par exemple, 
reconnue comme fondamentalement économique et monétaire, c'est pour le dénoncer et le fustiger. Le jugement de Pascal s'accorde parfaitement avec celui de Kant pour considérer l'ingratitude comme diabolique $^{27}$. Cette appréciation morale dont on pourrait montrer combien elle est elle-même solidaire de l'éthique des biens et de la sphère des paiements est un handicap pour qu'on y voie plus clair, en suivant Pascal, dans la destruction de l'idée de passion. Si étrange que cela puisse paraître et si loin qu'il ait poussé la conception structurale des passions qui est la première condition pour les faire voler en éclats, Pascal n'a pas cherché à définir les passions, sans doute parce qu'il est persuadé qu'elles ne souffrent que des définitions nominales, fragiles comme le sont les mots eux-mêmes, qui changent d'une langue à l'autre. C'est pourquoi je me tourne désormais vers Hume pour suivre sa thématisation des passions qui conserve et affine leur conception structurale ou, pour ainsi dire, nominale, mais qui pousse la réflexion sur ces points que Pascal s'était contenté de pratiquer, en redonnant paradoxalement une cohérence aux passions, les appelât-il des noms de Babylone ou de Sodome $^{28}$, par sa conception morale.

\section{Le moment humien}

Le point de vue de Hume, que l'auteur écossais se garde bien de qualifier de moral, puisque la morale même se construit avec des éléments affectifs qui lui sont hétérogènes ${ }^{29}$, retient tous les éléments dont nous avons remarqué, chez Pascal, qu'ils contribuaient à la dissolution de la notion de passion. Les passions n'ont pas de substance ; elles n'ont pas de consistance ontologique ; leur être n'est plus que celui d'une structure ou d'un ensemble de systèmes en constante évolution qui fait entrer en corroboration - selon son propre terme - la suite ininterrompue des sensations, avec leur réflexion de plaisir et de douleur, et la suite des idées qui leur correspondent de toutes sortes de façons avec elles-mêmes leur réflexion

dans la VIII lettre, quand il s'agit de «sauver la perte de l'un par le dommage de l'autre».

27 Dans le sens d'ailleurs où elle obtient l'unité des hommes par le jeu de leur division.

28 Voir la fin de la XIV e lettre des Provinciales.

29 A la différence de ce qui se passe chez ses prédécesseurs, Hutcheson et Shaftesbury : «Ceux qui ramènent le sens de la morale à des instincts originels de la nature humaine défendent la cause de la vertu avec une autorité suffisante, mais il leur manque l'avantage que possèdent ceux qui expliquent ce sens par une sympathie étendue avec l'humanité», Traité de la nature humaine, liv. 3, part. 3, sec. 6, Paris, GF-Flammarion, 1991. 
de plaisir et de douleur. Dans certains textes, différents des textes canoniques sur les passions que sont le Livre $2 \mathrm{du}$ Traité de la nature humaine et la Dissertation sur les passions, Hume distingue plus nettement une troisième ligne d'éléments qui permet la structuration des passions : celle qui est constituée, dans l'Essai sur la tragédie, par le langage ${ }^{30}$. Cette explicitation est intéressante, car elle renforce l'idée que les éléments symboliques scandent le sentir de la même façon que Berkeley l'avait établi pour la perception ${ }^{31}$, mais elle était déjà contenue dans la théorie de la double association, puisque les idées n'ont de sens chez Hume, comme chez Berkeley, auquel il se réfère explicitement ${ }^{32}$, que par des sensations singulières ou en voie de singularité, reliées à des mots qui en assurent une sorte de généralité. Le langage était donc présent au fondement de ce qui s'appelle passions dès les analyses du Livre 2 et de la Dissertation. D'autant que le découpage des passions n'a rien d'essentiel ni de substantiel : ce sont les mots qui fixent, un moment, une impression d'identité et d'extension bien délimitée. Mais la mouvance est toujours la plus forte et, à peine s'est-il tenu dans son identité précaire par un jeu de perspectives, que le système se défait déjà ; il faudra d'autres noms, plus ou moins adéquats, pour en marquer les états. C'est l'histoire et le cursus qui sont la vérité du système que l'on appelle des passions.

De plus, dans une philosophie qui ne reconnaît aucune substantialité au moi, mais qui tient son identité pour fictive, où les objets ne sont jamais construits que par fiction à partir des sensations, on conçoit que la passion perd aussi toute identité profonde, quoique ce soit par des notions affectives qu'un semblant d'identité revient aux choses ${ }^{33}$ et aux sujets $^{34}$. Sur ce dernier point, Hume ne se démarque pas nettement de

30 «L'impulsion ou la véhémence consécutives à la peine, à la compassion, à l'indignation, reçoivent une nouvelle direction relevant des beaux sentiments [ceux qui sont liés aux beautés expressives]. L'émotion prédominante de ces derniers saisit l'esprit tout entier, et prend la place des premiers sentiments ou du moins les imprègne assez fortement pour en altérer totalement la nature. Et l'âme, qui est en même temps soulevée par la passion et charmée par l'éloquence, ressent dans l'ensemble un mouvement puissant et d'un délice sans mélange", Hume, Les Essais esthétiques, part. 2, Vrin, Paris, 1974, p. 109.

31 George Berkeley, Nouvelle théorie de la vision, par. 46, dans Geneviève Brykman (édit.), CEuvres, Paris, PUF, 1985, vol. I, p. 224.

32 Du moins dans la section du Traité qui concerne les idées abstraites (liv. 1, part. 1, sec. 7).

33 Les passions sont le ciment du monde.

34 Est-ce un hasard si, juste après avoir compromis l'identité du moi à la fin du Livre 1 
Pascal, chez qui on peut trouver les mêmes éléments de scepticisme ; il devient, en revanche, plus radical que le janséniste, quand il s'avise de définir, non pas chaque passion - ce qui est impossible ${ }^{35}$ - mais la passion en général, le phénomène passionnel, qu'il reçoive un nom dans le détail ou qu'il n'en reçoive pas. C'est dans cet effort de définition que nous apercevons le franchissement d'un nouveau seuil dans le déclin de la notion de passion, encore qu'elle soit puissamment requise dans les explications des "sciences de la nature humaine», comme les appelle Hume, c'est-à-dire de celles qui traitent de la morale, de la politique, du commerce et de l'histoire. En effet, il y a deux définitions des passions chez Hume, qui ne s'explique jamais sur la cohérence qu'il paraît présupposer, bien gratuitement, entre elles dans l'usage très habile qu'il fait de l'une et de l'autre.

Dans la première définition qu'il donne des passions, au début du Livre 2, il insiste sur leur caractère "réflexif» et «secondaire» (secondary or reflexive) qu'il oppose au caractère primaire et original (original) des impressions qui, sans intermédiaire, paraissent nous livrer les choses mêmes. C'est le rouge et le doux de telle chose qui me plaisent et il faut que ces deux sensations soient pour que j'éprouve du plaisir. Le plaisir est une impression d'impression; ce caractère «secondaire» ou de $n^{\text {ème }}$ degré est confirmé par le fait qu'une idée peut déclencher un plaisir ou une douleur. Un plaisir dont je prévois la jouissance est lui-même un plaisir. Par là, Hume retient du mouvement des passions la possibilité de constituer des degrés, comme on constitue des fictions de divers ordres à partir d'entités réelles. Ainsi il peut rendre compte des aspects phénoménologiques des passions, en particulier des plaisirs de plaisirs, requis par le goût ou le sens moral, ou des déplaisirs de plaisir, etc., selon toutes les combinaisons possibles. Mais il est une deuxième définition de la passion, qui insiste sur un tout autre aspect, aussi agissant que le précédent dans toute son œuvre, et qui, en dépit de son intérêt intrinsèque, ne laisse pas d'inquiéter en ce qu'elle se donne comme directement opposée à celle du début du Livre 2 puisqu'elle est «une modification originale d'existence» (an original modification of existence),

du Traité, Hume attaque le Livre 2 sur les passions par cette passion du moi par excellence qu'est l'orgueil ? L'orgueil donne imaginairement une cohérence à un moi dont la substantialité et l'identité ont perdu toute réalité ontologique à la fin du Livre 1.

35 Hume le dit en commençant sa structuration de l'orgueil et de l'humilité (Traité, liv. 2, part. 1, sec. 2) ; il le rappellera au début de son analyse de l'amour et de la haine, liv. 2, part. 2, sec. 1). 
dont il convient de parler en termes de force (force, strength) et d'impulsion (impulse). Par cette définition, la passion perd son caractère «réflexif» et devient, à sa façon, une sorte de donnée première, caractéristique qu'on avait cru réservée aux seules sensations. Sans doute trouvet-on quelque correspondance entre les deux définitions : d'un côté, le jeu des séries de sensations, d'idées et de réflexions qui leur sont liées suppose une force d'inertie et des forces d'attraction ; de l'autre, la notion de force est éminemment secondaire en ce qu'elle doit être construite, comme c'est le cas dans la physique de Newton. Il est clair que, par cette dynamique, Hume explique, dans une suite sans origine ni fin, toutes sortes de phénomènes d'inertie, d'attraction, de composition en arbalète ou en oblique de passions ou d'intérêts divers. Mais la simple juxtaposition des deux définitions, sans que leur articulation ne fasse jamais l'objet d'une réflexion systématique et générale, crée toutes sortes d'équivoques dans l'analyse, car on ne sait jamais à quelle définition il convient de se référer pour expliquer tel ou tel phénomène et selon quels critères doit s'effectuer cette répartition. Il n'est pas sûr que l'on puisse trancher, autrement que par parti pris, le caractère plus fondamental de l'une ou de l'autre définition. On peut balancer entre le primat de la dynamique sur la phénoménologie, en faisant des représentations et des aspects sentis le simple masque des forces, et le primat du phénoménologique sur le dynamique, en recherchant, à propos de toute force, de quoi elle est la force. À supposer même que nous tenions ces deux points de vue comme s'ils étaient de valeur explicative équivalente, il faudrait encore présenter les principes de leur articulation. En dépit de l'extrême virtuosité que déploie Hume pour expliquer chaque conjoncture particulière, on ne peut garder très longtemps la conception optimiste d'un complément des deux perspectives. Même en passant sur la contradiction de l'originarité et de la secondarité, on trouve d'autres incohérences qui justifient l'éclatement, puis l'abandon pur et simple, quoique beaucoup plus tardif, de la notion.

L'illusion explicative obtenue par Hume au moyen des passions dans les phénomènes historiques, sociaux, politiques, est rendue possible parce que les principes qu'il a forgés de sympathie et d'autorité sont beaucoup plus cohérents que les deux définitions précédentes qui ne font que paraître désigner le même être. Pour m'en tenir à quelques traits saillants, comment la notion d'impulsion pourrait-elle être rendue cohérente avec celle de réflexion ${ }^{36}$ ? La vivacité que l'on accorde au phéno- 
mène passionnel est-elle un caractère phénoménologique, déjà difficilement compatible avec la «secondarité» de la réflexion par rapport aux sources vives que sont les impressions de sensation, ou faut-il délibérément en faire un caractère dynamique lié à la vitesse ou à l'accélération d'un processus? La couverture par le même nom d'entités aussi différentes n'embrouille-t-elle pas les choses ? On protestera, si on connaît bien Hume, que, précisément, des noms différents couvrent la vivacité, selon qu'elle est phénoménologique (vivacity) ou dynamique (vividness) ; encore faudrait-il montrer l'articulation systématique de ces termes. En revanche, sur le plan des principes, la sympathie, par laquelle on se figure saisir ce que l'autre sent et s'imagine que l'autre exige de soi, dans certaines circonstances, que l'on éprouve certains affects, est parfaitement cohérente avec l'autorité qui impose ses figures affectives de telle sorte que l'on s'y conforme. Le jeu structurel des identifications et des introjections rend parfaitement compte du surgissement et de la circulation des affects, lesquels sont ressentis, par ceux qu'ils habitent et traversent, comme naturels alors même qu'ils ne le sont pas. Ce que la sympathie requiert pour fonctionner, c'est certes un jeu réflexif à partir d'une situation appréhendée apparemment de façon plus immédiate, mais c'est aussi un "point-butoir» pour le jeu réflexif tel que j'imagine, à un certain moment, qu'on attend certains affects de moi, comme un sentiment $d$ 'amour pour mes enfants si je suis père ${ }^{37}$. C'est là que l'on rejoint l'autorité, qui fournit socialement les figures affectives comme de grands schèmes qui canalisent la vivacité des passions. On voit ici que, pas plus que la sympathie ne se réduit aux aspects réflexifs auxquels on la croirait d'emblée exclusivement attachée, l'autorité ne se résume à la seule force, qu'elle s'efforce plutôt de canaliser, de mettre à distance, de ralentir. Comme la sympathie, l'autorité n'échappe sans doute pas à la dissolution et à l'érosion dynamique, mais elle y résiste par des effets de système et de structure qui, un temps, font obstacle au flux universel dont parle Hume dans les Dialogues sur la religion naturelle. Les passions sont donc ces systèmes provisoires acquis, dont la transmission est plus sûre que ce qui est transmis, qui paraissent rendre les groupes cohérents,

discours de la double relation de celui de l'impulse : «les principes qui régissent ici la transition des idées concourent ici avec ceux qui agissent sur les passions ; en unissant leur action, ils confèrent à l'esprit une double impulsion».

37 On notera que le reproche que l'amour manque est plus vif que le plaisir de le voir exister. Le reproche fait à l'autre de ne pas sentir est une excellente monnaie d'échange. Preuve que les affects ne servent jamais qu'à enclencher des circuits ; ils ont moins de positivité qu'ils ne servent à circuler. 
d'autant qu'on aurait grand tort de faire coïncider leur théâtre avec le psychisme de chaque particulier. Il n'y a pas lieu de tenir l'individu pour le seul centre passionnel possible, puisque son identité au cours du temps n'est autre que fictive.

Le malheur de l'explication par les passions plutôt que par les séries d'éléments ou d'événements plus élémentaires qui sont censés les constituer, c'est qu'elle donne lieu à des discours tout à fait contradictoires entre eux. Dans le Traité, s'interrogeant sur le clivage des classes sociales, qui devrait encourager l'envie et la guerre civile, Hume montre avec brio que le sentiment joue une fonction conservatrice en ce quel'on envie plus violemment quelqu'un qui nous dépasse de peu par le degré de fortune, le degré de compétence ou d'habileté, qu'une personne qui, sur les mêmes registres, nous dépasse prodigieusement. Dans ce dernier cas, le sentiment protège l'incommensurabilité des conditions ${ }^{38}$. Ma pitié pour les pauvres, si je suis riche, ne va jamais jusqu'à renoncer à ma condition. Et, réciproquement, sur le versant qui apparaît le plus dangereux, comme je ne me mets pas facilement à la place du riche si je suis très pauvre, je ne peux pas l'envier et je prolonge ainsi mentalement le clivage des classes en le rentrant. Mais, quand on lit l'Histoire d'Angleterre, on trouve un tout autre discours, en particulier sur la période de la révolution qui culmine à la mort de Charles $\mathrm{I}^{\mathrm{er}}$ : Hume insiste, pour expliquer cette exceptionnelle crise d'autorité, sur le caractère stéréotypé des passions : mécontentement lié aux impôts estimés trop élevés, impression d'être méprisé, identification aux députés-vrais ou faux martyrs, phénomènes qui enclenchent et réenclenchent, quels que soient les événements, la même spirale de la guerre civile. Comment se fait-il que, dans des situations comparables d'inégalité, les passions enclencheraient la conservation de ces situations, d'une part, leur destruction ou leur résolution, d'autre part ? Cette contradiction apparente laisse au moins soupçonner que la valeur explicative des phénomènes sociaux et politiques par les passions est insuffisante et suspecte.

Par sa référence aux passions, Hume se donne trop de schèmes explicatifs, puisqu'ils se concurrencent jusqu'à la contradiction. Dans le premier cas du clivage bien tempéré, il fait jouer une loi affective qu'il a établie précédemment : la passion est moins un système ou ce qui accompagne un système qu'un moment vif du développement de celui-

38 Hume montre comment un sentiment a tendance à se renforcer en multipliant son jeu de réflexions et en développant toutes sortes de relations. La distance sociale se fait volontiers distance physique. Voir sur ce point le Traité de la nature humaine, liv. 2, «Les Passions», Paris, GF-Flammarion, 1991, p. 228. 
ci qui s'y substituerait. L'élément dynamique vif parait prendre la relève du système dans son ensemble ${ }^{39}$. Ainsi un sentiment de distance incommensurable vient-il accomplir vivement la relation sociale d'inégalité et en quelque sorte se substituer à elle. Dans le second cas, entre en jeu un autre aspect, qui est proprement une identification obsessionnelle à des affects extrêmement stéréotypés, auxquels personne ne croit, mal fondés dans les faits qu'ils paraissent accompagner, mais qui n'en jouent pas moins le rôle d'une référence extrêmement stable, qui ne s'effritera que lorsque la structure politique, sinon économique, qui a donné lieu à leur schème tutélaire, aura volé en éclats. Hume n'invoque pas le même aspect des passions pour expliquer la situation d'inégalité paisible et une situation d'inégalité insurrectionnelle et révolutionnaire. L'identité du mot passion dissimule mal que l'on ne prend pas du tout en compte les mêmes choses et les mêmes causes dans l'un et l'autre cas; le moment vif dont nous avons parlé paraît authentique ${ }^{40}$; le second mouvement est au contraire profondément inauthentique, l'identification ne se faisant que très partiellement, quoique le mouvement historique s'accomplisse contre ce qui est senti ou indépendamment de ce qui est senti. Derrière les passions et leur apparente valeur explicative, $c^{\prime}$ est toujours autre chose qui est en jeu. La grande subtilité de Hume est d'avoir exprimé, en parlant de passion, le fait que les raisons politiques, économiques, sociales ne nous contraignaient pas directement, mais de façon seulement oblique ${ }^{41}$. A-t-il bien choisi toutefois sa notion pour l'exprimer?

Inspectant, dans sa Deontology, sous le titre de Hume's virtues, l'usage que Hume fait de la notion de passion, Bentham en contestera le bienfondé, en en dénonçant le caractère idéologique de la notion, tant qu'elle n'est pas réduite à ses éléments constitutifs que Bentham conçoit dans le plaisir et la douleur, et qu'elle n'est pas reconstruite selon des axiomes et par des dérivations qui nous permettent d'en comprendre le processus, sans être contraints de les saisir comme des données empiriques originaires qu'elles ne sont pas. Il faut casser le schéma classique par des moyens logiques, parvenir aux véritables atomes de la question, s'il en est, et comprendre à partir de là la façon dont les éléments affectifs

39 On voit le système fonctionner dans la Dissertation sur les passions, sec. 2, par. 5. Même thème, liv. 2 , part. 1 , sec. 6 , vol. I.

40 Il paraîtra à Rousseau beaucoup moins vif et profondément inauthentique.

41 Selon l'un des mots les plus usités par Hume dans le domaine politique et élevés au concept. 
entrent dans tous les autres phénomènes ${ }^{42}$. Mais avant de parcourir ce travail logique, qui est le véritable acte de décès de la notion de passion, il faut ouvrir une parenthèse pour considérer ce que nous avons appelé le moment cynique, sans lequel on ne comprendrait que partiellement le point de vue de Bentham.

\section{Le moment cynique}

Le moment cynique est une phase indispensable dans le déclin de la notion de passion; l'utilitarisme n'aurait probablement pas pu se constituer sans traverser ce moment. Il s'agit à la fois de dire que ce que nous appelons les valeurs morales n'est constitué que par un jeu de passions, et que le jeu est globalement la résultante d'intérêts personnels ou collectifs, qui se masquent plus ou moins habilement. Il faut dire que les moralistes avaient bien commencé et que l'on trouverait chez eux le maximum de cynisme s'il n'était compensé par l'affirmation de valeurs morales dont ils déplorent qu'elles ne soient pas scrupuleusement appliquées. C'est le cas de Pascal, qui établit la suffisance de la concupiscence pour établir une police et une justice et l'insuffisance de l'enseignement de Port-Royal pour vaincre la nonchalance ${ }^{43}$. J'aurais pu citer Mandeville qui montre, dans sa célèbre Fable, que les vertus publiques sont faites de combinaisons et d'affrontements de vices privés ${ }^{44}$; la leçon sera parfaitement entendue par Hume, d'abord, qui comprendra très bien que la morale est faite de pièces rapportées, dont aucune n'est par elle-même morale ${ }^{45}$. Elle sera ensuite entendue de Kant qui compren-

42 Je laisse délibérément de côté les aspects utilitaristes dont Bentham souligne l'intérêt chez Hume. Certains textes moraux sont de purs calculs. Qu'on lise, par exemple, l'essai sur le suicide, dont le nerf est le suivant : je ne suis pas tenu de faire un faible bien pour la société si c'est au prix d'un grand mal pour moi-même. Dès lors je puis me retirer du circuit des paiements ; car, «lorsque je me retire complètement de la société, puis-je être lié plus longtemps ?».

43 Les Pensées, frag., p. 59 : «Les enfants de P.R. auxquels on ne donne point aiguillon d'envie et de gloire tombent dans la nonchalance».

44 La page de titre de la deuxième édition de 1714 était on ne peut plus explicite : La Fable des abeilles ou les vices privés font le bien public, contenant plusieurs discours qui montrent que les défauts des hommes, dans l'humanité dépravée, peuvent être utilisés à l'avantage de la société civile, et qu'on peut leur faire tenir la place des vertus morales.

45 L'un des chapitres les plus paradoxaux du Traité de la nature humaine, de ce point de vue, est celui qui porte le titre Moral distinctions deriv'd from a moral sense (liv. 3, part. 1, sec. 2), qu'on prendrait bien à tort pour la position personnelle de Hume ; la thèse de l'auteur est plutôt que le sens moral ne peut jamais exister avec l'autonomie que Hutcheson et Shaftesbury lui accordent. 
dra que les valeurs morales sont mieux réalisées par le jeu des intérêts et des passions que par une incertaine et toujours douteuse mise en œuvre de l'impératif catégorique ${ }^{46}$. Mais aucun ne va jusqu'à dire que la morale n'est que ce jeu d'intérêts et de passions qui nous apparaît illusoirement ou de façon inversée : Kant ne discrédite pas l'impératif catégorique au nom de la nature et de l'histoire ; Hume maintient l'illusion du sens moral; Mandeville distingue la morale des forgeries sans lesquelles la société ne pourrait pourtant exister et Pascal décrit la situation politique du point de vue de la charité.

Le seuil du cynisme est franchi lorsque les valeurs morales n'apparaissent plus que comme des leurres et des alibis du mouvement qui en constitue l'équivalent et qui est seul réel. Le cynisme est dans la réduction au «ne que». "Ce qui paraît générosité $n$ 'est souvent $q u$ 'une ambition de pensée qui méprise de petits intérêts pour aller à de plus grands». Les Réflexions ou sentences et maximes morales de La Rochefoucauld permettent cette lecture ; la permettent mieux encore les œuvres de Sade qui conseillait à chacun de n'être pas assez fou pour croire que ceux qui agissent par impulsion bienveillante sont meilleurs que ceux qui sont ouvertement intéressés : «Le sentiment de la vertu n'est qu'un sentiment vil et intéressé qui semble dire : "Je te donne pour que tu me rendes».» Ce qui nous renvoie au circuit des paiements, qui donne la clé de la morale, dont on peut sans doute agrandir le cercle qui ne tend qu'à cet élargissement, mais dont on ne sort jamais, quoiqu'un certain nombre de morales, qui préconisent le dépassement de l'éthique des biens, feignent de nous en donner l'espérance ${ }^{47}$.

On ne dépasse pas si facilement le «fait total» du circuit des paiements; on a beau soutenir, avec Kant, que les personnes ne nous sont pas chères comme le sont les marchandises et les choses, puisque les premières ont une valeur infinie que les autres n'ont pas ; en réalité, chaque acte moral a son prix. Bentham l'indique de deux façons qui

46 Dans la fameuse Idée d'une histoire universelle au point de vue cosmopolitique, quatrième proposition. (Opuscules sur l'histoire, Paris, GF-Flammarion, 1990, p. 74-76).

47 Si l'on accorde la distinction élaborée par Lacan dans le Séminaire VII (Paris, Seuil, 1986), il faut opposer aux éthiques des biens, qui sont les plus ordinaires, l'éthique de la loi (qui est celle de Kant) et l'éthique du désir (tel que Lacan le comprend). Par le biais de la dette, on comprend que l'éthique des biens n'est jamais que l'envers de l'éthique des devoirs. On lit dans L'Utilitarisme de John Stuart-Mill : «Le devoir est quelque chose qui peut être exigé d'une personne comme on exige le paiement d'une dette» (Paris, Félix Alcan, 1925, p. 130). Le sens moral est fondamentalement un sens de la redevance. 
peuvent choquer les belles âmes ; d'abord en contestant qu'il y ait des actes et des motifs désintéressés ${ }^{48}$ - ce qui est une façon un peu provocatrice de dire qu'on ne sort jamais du circuit des paiements, qu'il n'est pas en notre pouvoir de le vouloir et, moins encore, de le faire. Le désintéressement n'est jamais qu'une complication de l'intérêt que l'on n'aperçoit pas. Ainsi ce qu'on appelle industry n'est qu'une course après l'argent qui ne s'avoue pas et qui se donne comme amour $\mathrm{du}$ travail. Ensuite, Bentham fait ressortir, de façon très directe, que chacun a en quelque sorte le prix de son sens moral lorsqu'il a le choix entre s'acheter un plaisir et donner à un pauvre, par exemple ${ }^{49}$. Il ne faut pas opposer les valeurs du marché aux relations morales; on ne le fait qu'en payant sa bonne foi d'hypocrisie. Nous avons déjà remarqué que Pascal et Kant soulignaient les mérites de la concurrence et même les promouvaient plus ou moins ouvertement ; il n'empêche que ces auteurs ont continué à défendre leurs valeurs morales, non comme une rection sourde destinée à orienter les intéressées, mais comme les valeurs qui devraient seules exister ; ce qui est proprement verser dans $l^{\prime}$ hypocrisie. Ils ne croient pas à ce qu'ils veulent et n'agissent pas comme ils pensent.

Il y a plus : en voulant dissocier la valeur des personnes du prix des choses $^{50}$ et en rendant incommensurable la première au second, tout se passe comme si on assignait un prix infini aux personnes et rendait impossible par avance toute réparation (par le châtiment ${ }^{51}$, par l'assurance, par l'héritage ou que sais-je ?), voire tout travail du deuil. Or n'y a-t-il pas une sagesse à poser et reconnaître loyalement que tout malheur, toute destruction personnelle par exemple, se trouvent au bout du

48 Dans les Observations (par. 2) qui suivent la Table des ressorts de l'action; dans Bowring (édit.), The Works of J. Bentham, Edimbourg, W. Tait, 1843, reprint 1995, vol. I, p. 211-212.

49 « $\mathrm{Si}$, ayant une couronne en poche et n'ayant pas soif, j'hésite entre acheter une bouteille de bordeaux pour me désaltérer moi-même, ou la dépenser pour donner le moyen de vivre à une famille que je vois sur le point de périr faute d'assistance, tant pis pour moi à la longue : mais il est clair qu'aussi longtemps que j'ai continué à hésiter, les deux plaisirs de la sensualité, d'une part, et de la sympathie, d'autre part, auraient exactement pour moi la valeur de cinq shillings, seraient exactement égaux pour moi». Dans Élie Halévy, La Formation du radicalisme philosophique, Paris, PUF, 1995, vol. I, p. 307.

50 C'est, par exemple, le cas de Kant dans les Fondements de la métaphysique des mœurs, Paris, Vrin, 1992, p. 112-113.

51 La Théorie des peines criminelles de Bentham envisage nettement la punition dans un système de gains et de dépenses. 
compte compensée ? Il y a, dans le cynisme, une sagesse profonde, qui est celle-là même de l'homme ordinaire dont parle Hume ${ }^{52}$ qui s'occupe plus de ce que sera le destin des membres de sa famille après sa mort que de son salut post mortem. À trop invoquer l'incommensurabilité de la dignité de la personne, on enquiert le soupçon de vouloir simplement, quoique subrepticement, faire monter les enchères. La vérité du cynisme, c'est le fait total qui broie implacablement les valeurs et les rapporte impitoyablement les unes aux autres, sans que le moralisme, qu'il soit sympathique, sentimentaliste ou rationaliste, ne puisse y faire exception. Le pacte du paiement et de la souffrance ne se pose pas du tout dans les termes de l'infinitisation de la personne par rapport à la finitude des choses ; mais dans quels termes se pose-t-il ? Je suivrai désormais le fil conducteur de l'utilitarisme benthamien, qui servira au passage de lacet des passions.

\section{Le moment benthamien}

Je voudrais partir d'un fragment de Chrestomathia, livre de réflexion sur l'éducation utilitariste, dans lequel on voit les passions se décomposer à la fois sur un registre verbal et sur le registre pénal et économique des paiements. "Quant à la suffisance, la vanité et l'orgueil, «self-sufficiency, vanity and pride» auxquels on pourrait ajouter d'autres mots de pareille estampille, ils constituent un ensemble de termes sentimentaux «sentimental words», qui a pour effet de faire flotter autant de généralités vagues et indéterminées ; autant d'idées qui, aux yeux de celui qui prend la peine de leur trouver une forme déterminée, s'évanouissent et ne laissent derrière elles rien d'autre que les lettres et les sons que nous leur avons associés. Du côté des individus dont il s'agit, la suffisance, la vanité et l'orgueil, toutes ces faiblesses, à supposer qu'elles se manifestent, trouveront, dans l'esprit de ceux qu'elles rencontrent - ceux avec lesquels les circonstances conduisent à entrer en relation - des principes de résistance sous forme d'amour de soi et d'orgueil défensif "self regarding affection and defensive pride», par lesquels toute transgression des lois sociales trouvera son châtiment éventuel ; et, dans la crainte qu'elle se produise, un frein, une vérification, $a$ check [le mot commence à prendre un sens économique en 
anglais $\left.{ }^{53}\right]^{54} \gg$. Ce texte, rendu assez obscur par ma traduction, n'en est pas moins capital pour le problème qui nous occupe : les passions sont traversées par l'analyse comme des signifiés évanescents jusqu'à ce qu'elle atteigne les signifiants qui sont les points résistants de l'affectivité ; de plus, l'essentiel des passions tient dans un rapport aux lois sociales, qu'on entende par là les lois juridiques ou les lois économiques. Comment en est-on venu à une pareille décantation où les phénomènes passionnels perdent toute consistance autre que linguistique ou nomologique ? Il faut, pour comprendre l'intérêt de ce texte, qui pourrait passer assez inaperçu dans cette publication de 1817 probablement écrite aux environs de 1815, le mettre en relation avec quelques fragments tirés d'autres œuvres.

Le premier, écrit postérieurement en 1828, et peut-être le plus fondamental, car on y voit que Bentham ne se trompe pas de cible, est l'attaque, conduite sous le titre de Hume's virtues, de la notion humienne de passion. Après avoir salué chez Hume la plus importante distinction qui ait été faite entre les impressions et les idées, manifestant par là son intérêt pour la double association, par laquelle Hume rendait compte des passions, Bentham tempère son admiration par une critique et une remarque. La critique, c'est que Hume n'a pas vu la connexion qui existe, en général et non pas seulement dans des cas particuliers, entre la passion, le plaisir et la douleur ${ }^{55}$; or «plaisir et douleur constituent la seule clé pour ouvrir la serrure. Vous pouvez vous y essayer autant que vous voulez : vous ne $l^{\prime}$ 'ouvrirez que par cette clés ${ }^{5} »$. La remarque est qu'il convient de traiter une passion, non pas comme quelque chose d'existant, mais comme une entité fictive, a fictitious entity. Il faut désormais considérer la liaison de ces deux observations. Toute passion doit pouvoir être réduite à une certaine organisation de plaisir et de douleur ; plaisirs et douleurs sont

53 Voir le mot check dans The Oxford English Dictionary, 2d ed., Oxford, Clarendon Press, 1989, vol. III, p. 70, 16a. Cette remarque serait assez gratuite si Bentham n'affectionnait la proximité du vocabulaire économique, voire bancaire. Le mot stock, qui signifie à la fois réserve et capital en est une illustration ; import en est une autre.

54 Chrestomathia, Matin J. Smith et Wydham H. Burston (édit.), Oxford, Clarendon Press, 1983, p. 46.

55 On voit bien le passage du Traité auquel Bentham accroche son commentaire : "Les douleurs et les plaisirs corporels, qu'ils soient sentis ou conçus par l'esprit, sont la source de multiples passions ; mais ils naissent originellement dans l'âme ou dans le corps, comme il vous plaira de dire, sans aucune pensée ni aucune perception qui les précède» (liv. 2, part. 1, sec. 1).

56 Deontology, Amnon Goldworth (édit.), Oxford, Clarendon Press, 1983, p. 350. 
les éléments, les atomes ${ }^{57}$ des processus affectifs. Il importe au philosophe ou au savant de savoir partir du plaisir et de la douleur pour constituer ces processus dont les lois sont linguistiques, juridiques, économiques et, dans la mesure où aucune de ces structurations n'est stable, historiques. Tant qu'on prend les passions pour des données empiriques indécomposables, on est dupe de leur pseudo-simplicitée ${ }^{58}$, qui masque des réalités juridiques, sociales, économiques ; il convient donc de les analyser jusqu'à leurs éléments et de considérer pourquoi elles sont construites ainsi et paraissent nous intimer tel ou tel comportement. Il en est de ces passions, que nous pouvons fort bien ressentir, comme de certains mots qui sont "misexpressive» et ne nous rapportent que de travers à leur référent apparent ; ainsi les passions nous font-elles considérer faussement un certain nombre de circonstances, dont elles sont le «messentir» - si l'on nous accorde ce néologisme - ; nous ne pouvons nous en rendre compte sans effectuer un travail théorique spécifique.

Le caractère élémentaire du plaisir et de la douleur ne doit pas nous tromper ; les opérations qui les prennent pour objets de construction ne sont pas de simples sommes ou soustractions arithmétiques. Elles les mettent en rapport très indirectement ; on le voit bien quand Bentham parle de l'autorité du plaisir et de la douleur plutôt que de leur force. Le plaisir et la douleur ne déclenchent pas de comportements comme un choc déclencherait un mouvement en mécanique ; ils n'agissent que comme fondement de motifs d'action. Ce qui ne veut pas dire - il faut y prendre garde - que les calculs de plaisir et de douleur sont rendus dès lors impossibles. Ils sont simplement plus difficiles et plus indirects, comme on le voit dans les calculs bayesiens, qui évaluent les chances d'avoir raison quand on fait telle ou telle estimation de probabilité, et dans les formulations de lois économiques qu'ils permettent. Mais, avant d'entrer dans le détail de lois que Bentham n'a guère écrites en caractères mathématiques, puisqu'il en est resté aux réquisits de leur écriture, et quoiqu'il ne se soit pas trompé sur les lieux de possibilité du calcul, il faut considérer comment il est passé des positions les plus élémentaires de plaisir et de douleur aux lois juridiques, économiques et linguistiques,

57 On trouve cette notion d'atomes, sinon comme s'appliquant aux plaisirs et aux douleurs, du moins à certains de leurs paramètres, comme la durée, qui se laisse assez bien décomposer en atomes.

58 Hume invoque la simplicité des passions pour se justifier de ne pas les définir singulièrement. Voir Traité, liv. 2, part. 1, début de la sec. 2 et part. 2, début de la sec. 1. 
qui se substituent au discours des passions au moyen d'axiomes de la pathologie, qui sont les véritables instruments théoriques qui permettent de transformer les plaisirs et les douleurs, fussent-ils des objets d'autorité plutôt que des constituants physiques ${ }^{59}$, en lois articulées entre elles.

Il n'est certes pas question d'entrer dans le détail de ces axiomes de l'affectivité (car il faut entendre pathologie comme se référant à l'affectivité plutôt qu'à la maladie) ; on trouve plusieurs esquisses de ce travail dans les œuvres, en particulier dans les Pannomial Fragments réunis dans le volume III de l'édition de Bowring ${ }^{60}$. Il les définit comme les propositions les plus communément applicables par lesquelles on peut établir les différentes occurrences selon lesquelles le plaisir et la douleur se produisent dans l'esprit humain ${ }^{61}$. C'est ainsi que Bentham pose que «le plaisir, qui résulte, chez une personne, du spectacle de la douleur subie par un autre n'est jamais aussi grand que la peine ainsi soufferte ${ }^{62}{ }_{\Perp}$; que la perte d'un avantage est toujours plus cruellement ressentie que le gain du même avantage ne fait plaisir ${ }^{63}$; que, si l'accroissement du bonheur est fonction de l'accroissement de la richesse, cette règle atteint vite ses limites, car on ne peut pas doubler, tripler ou quadrupler son bonheur aussi facilement que ses richesses : ce qui deviendra, exprimée en caractères mathématiques, sur le terrain de l'économie, la loi de Sidgwick, celle de l'utilité marginale décroissante ${ }^{64}$. Il ne s'agit là que d'échantillons

59 La «phraséologie physique», comme l'appelle Bentham est dénoncée comme telle, mais aussi estimée inévitable par l'auteur de la Deontology : «Nous n'avons pas moyen de trouver à exprimer cela, dit-il au milieu de sa critique des passions chez Hume, si ce n'est par une «physical phraseology»» (p. 350).

60 Pannomial Fragments, vol. III, p. 224-230.

61 Pannomial Fragments, vol. III, p. 224.

62 Pannomial Fragments, vol. III, p. 225.

63 Stark, (édit.), Jeremy Bentham's Economic Writings, Londres, G. Allen \& Unwin LTD, 1952 (vol. I et II), 1954 (vol. III), 3 vol., vol. I, p. 359 : «A loss thus falling upon one part to equal amount occuring to an other part. Yes, to equal nominal amount : but in no point of view is the gain equivalent to the loss. It fails of being so even in point of arithmetical effet [ 5 taken from 20 , takes away $1 / 4$ : added to 20 , it adds only $1 / 5$ ] : but were it in this sense altogether equal it would want a good deal so in point of moral or, to speak more precisely, in point of pathological effect : in regard to the effect it has upon the feelings of individuals». Vol. I, p. $365:$ «Such is the constitution of human nature, that the enjoyment resulting from a gain is never equal to the suffering resulting from a loss».

64 Cette loi énonce que, lorsque la quantité consommée d'un produit augmente, l'utilité marginale de ce produit (c'est-à-dire l'utilité supplémentaire ajoutée par la dernière unité) tend à diminuer. 
prélevés dans un ensemble plus considérable d'axiomes ; on pourrait aussi certainement trouver chez Bentham des axiomes pathologiques plus fondamentaux encore, par exemple : que la douleur est toujours plus «réelle» que le plaisir, que tout paiement s'effectue plus fondamentalement en souffrance qu'en plaisir.

Il me suffira de noter que, s'il ne l'a pas atteinte, Bentham a cherché l'exhaustivité des axiomes et qu'il a eu, par là, l'idée d'un système général des fondements des sciences que l'on appelait encore «de la nature humaine». Le jeu du plaisir et de la douleur, des axiomes de la pathologie, des propositions qui en résultent et qui ne sont autres que les lois plus ou moins fondamentales de ces sciences humaines et du droit, démantèlent complètement les anciennes façons de structurer les passions, en discréditant non seulement leur organisation mais jusqu'à leur notion même. On conçoit dès lors que le problème qui se posait à Bentham n'était pas seulement de produire les lois qui «ignorent» les passions, ce qu'il fallait néanmoins faire à partir de leurs signes, mais aussi de les exprimer dans l'ancien système de passions que la langue ordinaire a conservé, en dépit du discrédit de leur concept. Le problème n'était d'ailleurs pas seulement d'expression; certes, il a bien fallu, pour poser l'axiomatique pathologique ${ }^{65}$, bousculer les taxinomies et nomologies ordinaires, mais il ne faudrait pas croire que l'on puisse rompre une fois pour toutes avec les anciennes façons de penser : elles restent longtemps en tension avec l'avancée et l'organisation conceptuelles de l'axiomatique. Cela ne veut pas dire que la pensée rigoureuse reste tributaire des affects de la langue courante, mais ses objets ne s'effectuent qu'en rapport avec elle. La substitution n'est jamais parfaite et ne peut l'être. Il y a plus : comme le langage scande, chez Berkeley, les séries perceptuelles, et nous illusionne en nous faisant croire à une transcendance des objets par les combinaisons de ces séries, il en va de même chez Bentham quand il repère que le langage scande les passions et nous fait interpréter faussement les séries qu'elles paraissent organiser, alors que ce sont ces séries qui, en réalité, fondent les passions. Elles sont l'interprétation, par les langues vernaculaires, de règles et de lois qu'elles paraissent structurer, mais qui, en réalité, fonctionnent sans elles, puisqu'elles les produisent comme masques de leur propre fonctionne-

65 L'expression d'axiomatique me semble légitime en ce que Bentham est parfaitement conscient des enjeux de l'usage des axiomes et se réfère avec beaucoup de précision à ce qu'en fait Euclide (par exemple au par. 2 de The Psychology of Economic Man). [Nous citerons désormais ce texte par l'abréviation PEM, suivi du $\mathrm{n}^{\circ}$ de la page du volume III des Jeremy Bentham's Economic Writings]. 
ment. Il faut regarder cette articulation de plus près en insistant sur la logique des paiements.

Bentham commence par remarquer que, lorsqu'ils échangent des marchandises et des services, ce sont, en réalité, des plaisirs et des douleurs que les hommes échangent sous couvert de l'argent, en s'efforçant de les rendre commensurables et en parvenant à les ployer à une mesure commune $^{66}$. Les lois économiques, sociales, ne sont pas des lois de choses, comme elles peuvent le paraître par leur allure physique ; elles sont les lois de nos appréhensions et de nos appréciations des choses, masquées par la nécessité d'échanger des choses et des services. Mais, en retour, «l'argent est l'instrument de mesure de la quantité des peines et des plaisirs. Ceux qui n'acceptent pas la précision de cet instrument «the accuracy of the instrument» doivent découvrir un instrument qui serait plus précis ou faire leurs adieux à la politique et à la morale ${ }^{67}{ }$. Il faut comprendre comment Bentham en vient à une telle affirmation.

D'abord, il s'agit d'une affirmation de méthode ; car Bentham multiplie les précautions pour qu'on n'aille pas s'imaginer mesurer les quantités de plaisir et de douleur comme des quantités de poids, d'étendue, de chaleur ou de lumière ${ }^{68}$. On peut bien doubler une somme $d^{\prime}$ argent, on ne sait pas ce qu'est un plaisir deux fois plus grand ${ }^{69}$. Ainsil'idée de Bentham n'est pas d'exprimer directement les plaisirs par de l'argent ; elle est d'exprimer par l'argent le style de rapport que les hommes entretiennent avec le plaisir et la douleur. La loi de l'utilité marginale décroissante, que Bentham a énoncée de façon qualitative seulement, indique parfaitement la différence de fonctionnement entre l'acquisition de marchandises et de services et l'acquisition de plaisirs ; elle peut même donner la mesure de

66 Défense de l'usure, dans Mélanges d'économie politique, Paris, Guillaumin et $C^{\circ}, 1848$, vol. II, p. 511 : «Smith et tous les économistes qui l'ont suivi ont enfin démontré que ce que l'on empruntait et ce qu'on prêtait n'était point de l'argent, mais bien ce qu'il pouvait procurer; que, dans les cas où les transactions de cette nature se faisaient sous la forme d'une somme d'argent, cette somme n'était qu'un intermédiaire, un acte de transport (transfer) pour ainsi dire, qui faisait passer d'une main dans l'autre les capitaux que le propriétaire ne jugeait pas à propos d'employer lui-même ; qu'enfin le loyer que payait l'emprunteur n'était pas le loyer d'une somme d'argent, mais bien celui des objets qu'il se procurait avec cette somme et, qu'en conséquence, cette locution populaire, intérêt de l'argent, était radicalement fautive».

67 Stark, vol. I, p. 117. Idem au par. 25 de The Psychology of Economic Man, vol. III, p. $437-438$.

68 PEM, p. 441.

69 PEM, p. 442. 
cet écart. Ainsi, en analysant la façon dont le plaisir et la douleur s'organisent autour de l'argent, on peut se servir à rebours de l'argent pour décrire et expliquer les complexes de plaisirs et de douleurs, et constituer de la façon la plus fine qu'il se peut le jeu des plaisirs et des douleurs. Bentham réserve un sort particulier à la passion de l'intérêt pécuniaire pour comprendre le fonctionnement des autres passions ${ }^{70}$.

Par cette technique de renversement ${ }^{71}$, il parvient à constituer le plaisir et la douleur en concepts à multiples dimensions ${ }^{72}$, parfaitement exprimables par l'argent. Une somme d'argent dont je puis profiter tout de suite n'a évidemment pas la même valeur que la même somme $\mathrm{d}^{\prime}$ argent dont je puis profiter seulement probablement, quatre ou cinq ans après ; il est normal que le prêt de cette somme qui m'est versée me coûte quelque chose. Il faut, pour que le débiteur et son créancier soient à égalité, que celui qui utilise aussitôt la somme verse à l'autre des intérêts. La probabilité virtualise la somme d'argent, qui perd son identité lorsqu'elle est projetée dans l'avenir ; elle ne peut retrouver quelque équivalence avec une somme réelle qu'en étant augmentée, lors du remboursement, dans certaines proportions. Il en va de même du plaisir qui, différé, n'a pas la même valeur que sur le champ. Il y a un prix de l'argent ; il y a un plaisir du plaisir ou une douleur du plaisir, etc., en une sorte de jeu réflexif, dont on peut distinguer les ordres et qui a sa précision dans tous les secteurs de l'existence où on l'envisage. Les prix ont beau être des fictions : les fictions aussi ont un prix.

Il ne sert à rien de dire qu'on ne parvient pas à donner une mesure exacte de ce jeu affectif ; la réalité est que toute société y parvient ; chacune parvient aux commensurabilités nécessaires ; chacune crée peut-être, par ces sortes de violence, des souffrances, mais elle peut aussi les rectifier sans que les dimensions du plaisir sur lesquelles on agit

70 PEM, p. XXIII.

71 Qui est très exactement celle des problèmes de mathématiques dits inverses et dont les philosophes, quoi qu'ils disent par ailleurs de l'application des mathématiques en philosophie, ont fait usage, comme c'est le cas de Kant dans la Critique de la raison pratique.

72 C'est le mot que Bentham utilise. (PEM, p. XXIX). Dans le texte sur Le Calcul des plaisirs et des peines, traduit par Halévy en appendice $2 \mathrm{du} \mathrm{I}^{\mathrm{er}}$ volume de La Formation $d u$ radicalisme philosophique, Bentham souligne qu'il «emprunte aux mathématiciens la dénomination de «dimension" et rappelle que «Dimension» vient de «dimetior», «mesurer». On trouve, dans ce texte, cette phrase remarquable que «l'on peut parler de l'étendue [qui a elle-même ses dimensions] comme d'une troisième dimension d'une portion de bonheur", s'articulant avec l'intensité et la durée». 
doivent être contemplées comme des données naturelles. L'utilitarisme, en dépit des allures qu'il se donne parfois par ses métaphores, est tout sauf un naturalisme ${ }^{73}: l^{1}$ équilibre fragile, facile à dérégler, constamment à refaire, qu'il envisage entre les plaisirs est toujours culturel. Il est l'affaire des lois civiles, pénales, du politique et de chacun qui œuvre dans sa sphère avec plus ou moins de responsabilité sociale. Aucune nature ne vient nous offrir clandestinement et implicitement des règles, en dépit de l'illusion que l'on en peut avoir lorsqu'on regarde en miroir notre propre œuvre qu'on refuse de reconnaître comme la nôtre.

Outre la probabilité envisagée de façon «subjective» comme fraction de certitude, il est d'autres dimensions qui, comme l'intensité, la durée, la proximité ou l'éloignement, sont relativement faciles à mesurer ; quant à la fécondité, à la pureté et à l'extension, qui se révèlent parfois plus délicates sous l'angle de la mesure, elles posent le problème, essentiel depuis Pascal et Hume, du «centrage» du calcul des plaisirs et des douleurs. Le système dans lequel fonctionne ce calcul et s'équilibre son jeu n'est pas donné à l'avance ; à supposer même que l'on fasse de l'individu le cadre où se ressentent plaisirs et douleurs, comme il arrive à Bentham de le dire $^{74}$, les limites du jeu n'en seraient pas éclaircies pour autant, ni rendues moins problématiques. Chaque événement ou phénomène que l'on analyse impose à la recherche son propre format. C'est ce flottement permanent sur le format qui fait croire à la naturalité des passions. En réalité, la passion est le produit d'un système en concurrence permanente avec d'autres systèmes; ille est calculable. Elle se résout en calculs et indique des centres à partir desquels se développent des calculs. À ceux qui objectent à Bentham que les hommes calculent moins qu'ils ne sont soumis à leurs passions, Bentham rétorque, dans sa Psychologie de l'homme économique, que «tous les hommes calculent, même les plus fous, avec plus ou moins d'exactitude» et il se reprend pour établir avec plus de finesse, car c'est le point d'appui des calculs et la possibilité de la commensurabilité qu'ils requièrent qui se trouvent alors problématisés et posés comme réquisits : «Passion calculates, more or less, in every man» : «La passion calcule plus ou moins en chaque homme $[. .$.$] ; selon$

73 Il est vrai que le début de l'Introduction des principes de la morale et de la législation est assez équivoque; mais on voit très vite qu'il s'agit de métaphores dont Bentham exige le dépassement. Bowring (édit.), The Works of J. Bentham, Edimbourg, W. Tait, 1843, reprint 1995, vol. I, p. 1.

74 On lit, par exemple, dans la Défense de l'usure, qu'«il est bien rare qu'un homme soit meilleur juge dans les affaires d'autrui que l'intéressé principal» dans Mélanges d'économie politique, vol. II, p. 532. 
les motifs qui les font $\operatorname{agir}^{75} \gg$. La société résulte d'un immense entrecroisement de calculs de tous ordres ; l'absurdité est de vouloir l'empêcher, comme lorsqu'un gouvernement s'avise, pour des raisons qu'il estime morales, de fixer le prix de l'argent ou quelque autre prix d'ailleurs. On ne décrète pas que des plaisirs et des douleurs se mettent indéfiniment en rapport les uns avec les autres, comment ils doivent le faire, selon quelle commensurabilité. Cette commensurabilité s'indique d'elle-même, sans qu'on ait à fixer intrinsèquement les bons et les mauvais plaisirs, et en ne référant jamais, quand on doit rendre compatibles ces plaisirs, le bon et le mauvais qu'au principe d'utilité, qui finalise l'action en fonction du plus grand bonheur pour le plus grand nombre.

Conformément à sa méthode d'articulation, non seulement des différents paramètres entre eux, qui structurent le plaisir comme une grandeur, mais des grandeurs entre elles et des instruments pour les calculer, qui faisait apparaître, dans le plaisir, tout ce qui en permettait l'approche mathématique, Bentham procède encore une fois à rebours pour montrer ce qui, dans l'expression mathématique, doit aux opérations commerciales. Il établit très judicieusement comment, derrière l'algèbre, fonctionnent des schèmes issus du commerce et autres opérations d'échange. Comment entendre qu'une quantité négative, c'est-àdire qui est moins que rien, puisse prendre une sorte de consistance qui la rend susceptible d'entrer dans des opérations, sans envisager sa cohérence à la façon d'une dette, qui est à la fois un appauvrissement radical, puisque ce quej'ai est inférieur à zéro, et un acte plein ? La fiction de la dette fournit le noyau - comme eût dit Desanti ${ }^{76}$ - de la compréhension des nombres négatifs. Et sans doute Bentham voit-il l'effet de ces noyaux ou schèmes clandestins dans la compréhension d'opérations autrement inintelligibles comme le produit positif de deux quantités négatives, quoiqu'il n'en dise rien explicitement; car l'interprétation qu'il trouve de cette opération aberrante se situe dans la présentation, comme un acte substantif et à part entière de la négation : "The taking of a negative quantity negatively destroys the very property of negation and is the conversion of negative into positive numbers ${ }^{77}{ }^{\prime}$. Ainsi en

75 PEM, p. XXIII.

76 La Philosophie : un rêve de flambeur esquisse une théorie du noyau, qu'il envisage sans doute trop, à notre goût, comme une sorte de corps fantasmé.

77 Chrestomathia, p. 379-380 : «Le fait de prendre négativement une quantité négative détruit la propriété même de négation et est la conversion d'un nombre négatif en nombre positif». 
va-t-il d'une dette et de sa suppression. Être en dette d'une dette peut être l'équivalent $d$ 'un avoir. On voit comment la symbolique des circuits d'échange que nous explorions sous-tend les mathématiques mêmes qui sont utilisées pour rendre compte de ces échanges ; car si Bentham est resté à l'orée des calculs dans lesquels il voyait pourtant la seule marque de probité de ce qu'il avançait ${ }^{78}$, les économistes et sociologues utilitaristes passeront bientôt à l'acte et parviendront à donner une expression mathématique et, pour ainsi dire, exclusivement mathématique, à ce que Bentham n'envisageait encore qu'en termes philosophiques.

$\mathrm{Ni}$ le lieu ni le temps n'étaient propices à un développement de ces calculs à l'orée desquels s'est prudemment tenu Bentham. Seules la théorie des jeux et les recherches mathématiques de points d'équilibre dans la recherche des intérêts des membres d'une société réaliseront, beaucoup plus tard, la vocation calculatoire de l'utilitarisme, mais ce sera en remettant profondément en cause l'hédonisme benthamien. En revanche, je voudrais, pour terminer, relever les points sur lesquels Bentham a marqué durablement quelque avantage sur de nombreux successeurs utilitaristes et qui expliquent ultimement, au moins dans la ligne que nous avons suivie, le déclin de la thématique des passions. Ces points tiennent tous à l'élaboration de ce qu'on a pu reconnaître comme les éléments d'une théorie des fictions et dont ne se soucient pour ainsi dire jamais les utilitaristes modernes ; ce qui leur fait pourtant peut-être perdre un des sens de leur entreprise.

\section{La théorie des fictions et les passions}

Les utilitaristes modernes se passent radicalement des passions et avancent dans leurs calculs comme les géomètres de la fin du XIX ${ }^{e}$ siècle et du début du $X X^{\mathrm{e}}$ siècle, qui fondaient la géométrie parfois sans grand souci des types d'espaces anciens qu'ils ignoraient superbement. Les uns et les autres ne prennent guère en compte ce à quoi ils s'arrachent. C'est en ce sens que les utilitaristes de notre temps n'ont plus besoin de Bentham, qu'ils ne citent jamais et qui, sans doute parce qu'il faisait les premiers pas, se souciait davantage de contester ceux qui parlaient de passion que de se mettre réellement à calculer. Ce qui intéressait Bentham et nous intéresse encore avec lui, c'est la façon dont il prend le contre-

78 Deontology, p. 58 (A Table of Springs of Action : marginals), par. 631 : «Calculation the instrument of utility». Par. 632 : «Without calculation, the principle of utility might float useless in the sea of words with other phantoms of the imagination». 
pied de la manière ordinaire d'envisager les phénomènes sociaux, juridiques, politiques, embrumés dans les signifiés passionnels, pour esquisser des lois ou préparer leur terrain. L'effectivité de l'abandon de la notion de passion, ainsi que la conscience que l'on a travesti par son moyen l'intérêt général, prennent souvent plus de valeur, à ses yeux, que la production des concepts qui nous en délivrent efficacement. Tel est le lieu où travaille le théoricien des fictions.

Il peut bien faire avancer lui-même les concepts et établir les lois qui déterminent le véritable fonctionnement affectif ; il ne le fait pas sans observer comment ces lois sont arrachées aux façons communes de penser et de dire les pensées d'où il faut pourtant bien partir dans le travail de la conception et qui lui font cependant obstacle. Le langage ordinaire se moque bien de la vérité et de la philosophie ; presque tous les mots, presque toutes les formes syntaxiques représentent autant de dévoiements par rapport au concept. Le malheur - théorique et souvent pratique -, c'est que ces dévoiements empêchent que l'on y voie clair sur le véritable fonctionnement de la société, de l'économie, du droit et de la politique, quoiqu'ils soient, de ce fonctionnement, un précieux indicateur. Une théorie des fictions est, de ce point de vue, une théorie du schématisme entre la conception de ce qui se passe et qui tente de définir de vrais sujets et de vrais objets, d'une part, et, d'autre part, ce qui est senti de toutes sortes de façons et donne lieu à de faux objets et de faux sujets. Cette théorie du schématisme est d'autant plus importante que les concepts ne pourraient exister sans les mots et les propositions qu'il faut dépasser et corriger sans cesse à travers leurs mauvaises désignations et expressions. Il y a une illusion du concept, qui paraît fonctionner comme si le théoricien avait le choix des mots pour le dire et qui semble aller son chemin de façon autonome ; le discours des passions est sans doute faux, mais il est comme la seconde ligne qui nous permet de lire la précédente et d'en concevoir les propriétés révélatrices, inversantes et, par conséquent aussi, méconnaissables - car il est difficile de voir, sans un travail spécifique, dans un livre de jurisprudence ou d'économie, la réalité d'un traité des passions.

Bentham n'a certes pas théorisé la notion d'inconscient ; il ne la désigne pas ainsi, mais il en rencontre l'équivalent dans tous les ouvrages où il considère et dénonce le travail idéologique du langage ${ }^{79}$. L'inconscient n'est pas un discours obscur ; il est la modification qui, dans le discours

79 Par exemple dans le chapitre 3 de la $5^{\mathrm{e}}$ partie du Manuel de sophismes politiques dans Fragment sur le gouvernement. Manuel de sophismes politiques, Bruxelles, Bruylant / Paris, LGDJ, 1996, p. 356. 
même, rend méconnaissable ce dont on imagine parler. Si l'on se tourne vers le langage, on remarquera qu'il est énormément de choses qu'il nous empêche structurellement de dire et, par conséquent, qu'il voue à la non-fixation, qu'il dérobe à la maîtrise et au contrôle ; que l'on ne distribue pas, dans une langue, les mêmes objets que dans une autre ; que l'on peut dévoyer une structuration par une autre. On pourrait, à titre d'exercice, repérer la différence entre ce qu'on peut "payer» en français et ce qu'on "pay» en anglais ${ }^{80}$; on verrait aussitôt que le mot n'est pas scandé de même en chacune de ces langues et que l'accès au concept, qui est nécessairement linguistique, y est radicalement différent de part et d'autre. On imaginerait bien à tort que cela n'a aucune incidence sur le développement du concept ; les concepts appartiennent à une langue, fût-ce dans les conditions difficiles que j'ai pu décrire ; ils peuvent certes être transfusés dans une autre langue, mais ce n'est pas sans distorsions difficiles et coûteuses.

Ainsi les passions se sont évanouies le long des lignes où l'on cherche proprement le fonctionnement du social et de l'économique ; elles n'existent, ou plutôt on s'imagine qu'elles n'existent, que dans la différence entre les lignes où l'on tente d'établir la réalité des processus et celles où on se figure lire la réalité, qui importent toutefois pour que la conceptualisation puisse avoir lieu, quoiqu'elles ne soient qu'une interprétation fragile et évanescente d'un phénomène total où elles ne font que jouer leur partie. Elles sont aléatoires en comparaison des phénomènes auxquels elles croient se référer et leur nécessité est ailleurs, dans une logique qui est celle de la langue par laquelle elles s'expriment ; ce dont Hume avait pris conscience lorsque, après Aristote, qui traitait des passions dans sa Rhétorique, il donnait un style littéraire à chaque passion avant d'en construire la structure ${ }^{81}$.

Il est toutefois un point sur lequel Bentham s'est assez lourdement trompé ; ce n'est pas sur son "atomisme», que son caractère méthodique rend tolérable et finalement compatible avec une conception structurale du plaisir et de la douleur ; mais c'est sur le fait qu'il n'envisage pas de dépassement du plaisir et de la douleur, et qu'il traite ceux-ci comme s'ils étaient des réalités psychiques ultimes; ce qui est un préjugé ${ }^{82}$.

80 «One pays attention to a feat», en anglais, alors qu'en français «on [y] prête attention».

81 Nous avons dit quelques mots là-dessus dans l'introduction que nous avons écrite à notre traduction du Livre 2 du Traité de la nature humaine, Paris, GF-Flammarion, 1991, p. 18-19. Une étude plus systématique serait nécessaire sur ce point.

82 La psychologie moderne nous a appris à nous situer au-delà du principe de plaisir. 
Toutefois cette erreur ne saurait remettre en cause la dissolution de la notion de passion; cette dissolution paraît acquise pour la psychologie et la psychiatrie modernes, comme pour toutes les autres sciences de l'homme d'ailleurs, si on en excepte une certaine histoire de la philosophie, qui n'en finit pas de parler de passion, en omettant de décrire comment son objet est devenu périmé.

Il en va décidément des travaux sur les passions comme de ceux de Hilbert à l'égard d'espaces qui n'étaient plus les siens à la fin du XIX siècle, mais qui avaient été ceux qui rendaient possibles les théorèmes de Desargues et de Pascal. Nous savons pourquoi ces théorèmes sont faux et ils continuent de nous hanter pour des raisons autres que théoriques $^{83}$. De même, nous manquons de travaux pour décrire la disparition de la notion de passion, son mode particulier, le type de cicatrice qu'elle laisse par l'espace culturel constitué par le faisceau des lignes dont nous parlions ; peut-être, en les commençant, parviendrons-nous à avoir un tout autre rapport à cette notion dont nous ne savons pas faire notre deuil et qui, d'ailleurs, est peut-être constitutive de notre affectivité, toute périmée qu'elle soit et en raison même de cette péremption. Nous comprendrons ainsi beaucoup mieux l'attirance de nos contemporains pour le thème des passions, ce qu'ils cherchent à travers lui et de quoi ils parlent réellement, quoique de façon voilée ${ }^{84}$. Il n'est d'ailleurs pas sûr qu'il y ait eu quelque âge d'or de la notion où il fut possible de parler de passions sans arrière-pensée, comme si elles existaient. Comment un théologien catholique, un économiste, un juriste et un auteur de

Chacun connaît l'ouvrage de Freud, écrit en 1919-1920, qui porte ce titre, Jenseits des Lustprinzips, et qui condamne la psychologie benthamienne, sur ce point au moins, à la naïveté. Est-ce un hasard, d'ailleurs, si, dans cet ouvrage, voulant illustrer les cycles dans lesquels tourne la vie des hommes, de certains d'entre eux du moins, Freud prend l'exemple «des bienfaiteurs qui, après quelque temps, sont quittés dans le ressentiment par chacun de leurs protégés, si différents que ceux-ci puissent être par ailleurs, et auxquels il semble donc dévolu de vider la coupe amère de l'ingratitude" (Sigmund Freud, CEuvres complètes, vol. XV, André Bourguignon, Pierre Cotet et Jean Laplanche (édit.), Paris, PUF, 1996, p. 292) ? Bentham a vu l'équivalent du principe de plaisir, d'une part, les cycles de paiements décrits de façon répétitive, d'autre part, mais il n'a pas vu que la répétition pouvait être contradictoire avec le principe de plaisir et plus profond que lui : «la contrainte de répétition [...] nous apparaît comme plus originelle, plus élémentaire, plus pulsionnelle que le principe de plaisir qu'elle met à l'écart» (p. 294).

83 Pédagogiques ou esthétiques.

84 C'est-à-dire dont ils recherchent la géométrie, la dynamique, la probabilité, toujours en se servant des auteurs du passé. 
tragédies ont-ils jamais pu penser à la même chose en écrivant ce même nom ? Les notions n'existent jamais que dans d'interminables galeries sans début ni fin clairement assignables et ramifiées en un inextricable réseau ; leur identité est incertaine et ne dépend que de l'usage que l'on veut ou peut en faire. En enquêtant sur la fin de la notion de passion, nous ne recherchons certainement pas un événement réel ; nous voulons voir comment une notion se résorbe ou nous apparaît se résorber du point de vue que nous occupons. Qu'est-ce que s'éteindre pour une notion, alors même qu'elle reste proliférante par le titre des ouvrages qui s'intéressent à elle ? L'extinction est moins une notion qui existe en soi qu'une notion extrêmement relative, qui fait surgir, à un moment donné, un ensemble de textes, lesquels paraissent s'inscrire dans une suite qui, graduellement, fait disparaître tous les usages que l'on assignait à la notion, en les «remplaçant» par d'autres.

\section{Conclusions}

1. Les philosophes ont une prédilection pour les commencements, les naissances et les origines des notions ; ils n'en ont presque aucune pour leur déclin et leur disparition, qui, symétriques des précédents, sont presque aussi fréquents qu'eux. Ce refus de considérer la fin des concepts a un coût ; les philosophes paraissent se résoudre à rendre leurs concepts souvent étrangement boiteux, puisqu'ils sont nés et semblent dès lors voués à jouir d'une espèce d'éternité unilatérale ou hémiplégique. L'idéologie des événements fondateurs de pensée ne tient-elle pas de cette incapacité à penser la fin des notions dont on ne relève que le commencement ${ }^{85}$ ? C'est l'un des mérites des Fondements de la géométrie de Hilbert de nous apprendre à considérer des fins de concepts ; cette notion de fin est difficile car elle nous met en double porte-à-faux : par rapport au travail de création qui «remplace» la notion défaillante ; par rapport à la notion dont on traite, qui n'est pas lue dans

85 Cette idéologie est typique de la phénoménologie husserlienne, telle qu'on la voit à l'œuvre dans L'Origine de la géométrie : «[L'existence géométrique] a, depuis sa proto-fondation, une existence spécifiquement supra-temporelle et accessible, comme nous en avons la certitude, à tous les hommes et, en premier lieu, aux mathématiciens réels et possibles de tous les peuples, de tous les siècles, et ce sous toutes ses formes particulières". "Le théorème de Pythagore, toute la géométrie n'existent qu'une seule fois, si souvent et même en quelque langue qu'ils puissent être exprimés", Edmund Husserl, L'Origine de la géométrie, Paris, PUF, 1990, p. 179. 
le sens qui paraît lui donner vie. Il serait temps de substituer, à la semi-éternité des concepts, une temporalité plus complexe.

C'est un étrange destin que celui de la notion de passion si on le compare à celui de sa voisine, la notion de perception, par exemple, ou à celui d'un certain type d'espace. Lorsque Berkeley établit que les éléments symboliques - le langage - trament la perception dont ils permettent les objets et l'impression de transcendance, il n'abolit pas pour autant les représentations perceptives ; alors que Bentham, lorsqu'il se livre consciemment au même travail sur les passions que Hume et que Berkeley sur la perception, exhibant les soubassements symboliques de la notion, fait d'emblée une tâche beaucoup plus destructrice. La notion s'est simplement trouvée sauvée par la modestie de l'axiomatique benthamienne et par l'ambiguité de l'attitude de l'utilitariste à l'égard du plaisir et de la douleur ; ainsi s'est-elle placée entre deux morts, si l'on ose dire, donnant à la notion une étrange temporalité dans notre culture : être du passé auquel personne ne croit plus, alors qu'il fascine encore et se trouve à l'origine d'une profusion de traités sur des traités, puisque personne n'écrit plus au premier degré - si cela s'est jamais fait - de traité sur les passions. Il est temps de passer à la conscience de ce type d'écriture et d'engager une connaissance de la passion comme fiction, exactement comme Hilbert s'est avisé, il y a un siècle, que le bel espace de Pascal et de Desargues, celui de la perspective et des trois dimensions, n'était plus le nôtre.

2. Le deuxième point est une précaution : je n'ai pas voulu dire que seule la sphère des paiements - même entendue de façon élargie, comme je l'ai fait : après tout pacare veut dire autant pacifier ou apaiser que payer - permettait d'expliquer la résorption de la notion de passion. J'ai choisi ce simple fil pour considérer un phénomène beaucoup plus global, dont il faudrait examiner les aspects juridiques - A. Smith et Bentham s'y sont consacrés - , psychiatriques - cette recherche doit évidemment passer par Pinel et Esquirol ${ }^{86}$, Cabanis, Crighton -, histo-

86 Pinel, l'auteur du Traité médico-philosophique sur l'aliénation mentale qui paraît en 1800 ; Esquirol, auteur Des Passions considérées comme symptômes et moyens curatifs de l'aliénation mentale, thèse soutenue en 1805. Marcel Gauchet note que les moyens théoriques d'Esquirol «expliquent que [son œuvre] deviendra bientôt inintelligible au regard de la postérité : tout ce qu'il a sous la main, en effet, pour mener à bien l'explicitation-systématimation des indications sommaires données par Pinel, le seul langage disponible, c'est la psycho-physiologie stoïcienne des passions, réactivée et revisitée par le vitalisme montpelliérain. Un langage que les avancées de la physiologie et l'irrésistible montée en puissance de l'organe cérébral ne tarderont pas à disqualifier», Gladys Swain, Dialogue avec l'insensé. Essais d'histoire de la psychiatrie, 
riques - Hume avait commencé le travail contre les principes mêmes les plus apparents de sa philosophie - La précédente explication du déclin des passions reste donc extrêmement partielle ; elle est toutefois suffisamment consistante et développée pour inquiéter une thèse qui s'est répandue depuis une ou deux décennies et qui entend se servir de Mauss pour tourner les assertions de l'Essai sur le don à l'encontre de l'utilitarisme. J'avoue mal comprendre le fondement de cette thèse, qui défend le désintéressement contre la dénégation benthamienne, mais qui laisse de côté le point essentiel, qui est celui du fait total, lequel est évidemment présent et agissant dans la théorie de Bentham et permet de rejoindre une idée que les deux auteurs partagent : celle du cycle infernal dans lequel nous engagent tant les paiements que les dons. $\mathrm{Ne}$ passe-t-on pas à côté de l'essentiel, quand on oppose, chez Mauss, le don au paiement ${ }^{87}$ ?

3. Mais il est un point peut-être plus important que tous ceux que nous venons de soulever, tout simplement parce que ceux-ci dépendent tous de celui-là : rappelons-nous que la recherche que je viens de proposer très partiellement repose sur une hypothèse, celle du déclin d'une notion. Or je ne sais guère ce que signifie un tel déclin, d'autant qu'il n'est pas homogène d'une notion à l'autre; ne serait-ce que dans un secteur comme la physique, le phlogistique n'a pas décliné comme l'éther, qui connaît de brusques retours. Est-il possible de mesurer un déclin et à quels critères le reconnaît-on ? Il eût sans doute été raisonnable de $\mathrm{s}^{\prime}$ assurer du fait avant d'en tenter une explication, car je serais mis dans une étrange position si le fait pouvait être démenti. Il me faudrait avouer une précipitation et un manque de sagesse si l'établissement d'un fait restait toujours étranger à la détermination de ses raisons ; ce qui n'est certainement pas le cas des passions.

\section{JEAN-PIERRE CLÉRO}

Université de Rouen

précédé de À la recherche d'une autre histoire de la folie, par Marcel Gauchet, Paris, NRF Gallimard, 1994, p. XXXIV. C'est du moins l'indication d'une hypothèse qu'il est nécessaire de creuser.

87 Il est vrai que Mauss le fait quand il oppose au système du don l'économie de marché, mais l'idée selon laquelle «le don est à la fois ce qu'il faut faire, ce qu'il faut recevoir et ce qui est cependant dangereux à prendre " convient parfaitement au paiement, tel que nous l'avons décrit. Marcel Mauss, Sociologie et anthropologie, Paris, PUF, coll. «Quadrige», 1993, p. 249-250. 